\title{
Dynamics of Interaction of a Single Fiber with a Headset of a Sampling Drum
}

\author{
Yuldashev Jamshid, Umarov Akbarjon, Sarimsakov Olimjon (1) \\ Namangan Institute of Engineering and Technology, Namangan, Uzbekistan \\ Email: olimjon5008@mail.ru
}

How to cite this paper: Jamshid, Y., Akbarjon, U. and Olimjon, S. (2020) Dynamics of Interaction of a Single Fiber with a Headset of a Sampling Drum. Engineering, 12, 347-355.

https://doi.org/10.4236/eng.2020.126027

Received: March 28, 2020

Accepted: June 16, 2020

Published: June 19, 2020

Copyright (c) 2020 by author(s) and Scientific Research Publishing Inc. This work is licensed under the Creative Commons Attribution International License (CC BY 4.0).

http://creativecommons.org/licenses/by/4.0/

\begin{abstract}
The article presents an analysis of the dynamics of interaction of a single fiber with the sampling drum of a headset during pneumomechanical spinning. The regularity is investigated and the equation of the movement of fibers on the surface of the tooth of the sampling drum headset is obtained. It was found that the dependences of the movement of fibers along the tooth at different angles of inclination, the distribution of fiber tension along the arc, and the distribution of fiber speeds in the region of the sampling arc have an increase property. As a result of the analysis of the tension and speed of the fibers, it was found that with the increase in the sampling zone, the tension force and speed of the fiber increase.
\end{abstract}

\section{Keywords}

Fibers, Sampling, Angle, Friction Force, Friction, Speed, Radius, Fiber Complex

\section{Introduction: Formulation of the Problem}

In the discretization zone, the headsets of the sampling drum enter the beard of the clamped fibers. The amount of immersion of the teeth of the headset depends on the shape of the supporting surface to which the fibers are pressed by the headset. The teeth of the headset act on fibers that are within their reach, and those fibers whose relationship with the mass of the tape is less than the impact of the headset are pulled out of the feed product-the tape.

In this case, the interaction of the tooth of the headset with a beard occurs according to two schemes. In the first scheme, the tooth acts on the fiber with its front face, drawing it into motion. According to the second scheme, the lateral face of the tooth creates friction forces, which when the headset is immersed in the beard, cause a certain force in the fiber, which leads to partial straightening of the curved fibers. 
The fibers held by the rear ends in the clamp of the feed device are under the influence of friction forces and resistance to pulling fibers, which are in active interaction with the teeth of the headset.

In the process of interaction, one or several fibers are pulled out from the beard, which subsequently come into contact with each other and form a single complex. The separation of the fibers occurs during the implementation of mainly the second interaction scheme, where it occurs at a high frequency of exposure of individual teeth of the headset throughout the sampling area.

In this case, the headset fibers are transported from the region of high density beards in the region of low density. The orientation of individual fibers in the headset depends on their location in the beard before interacting with the teeth of the headset. If the fibers are at an angle to the direction of the headset, they can be elongated with the front edge of the tooth. Such fibers practically do not parallelize, tend to form knots. Taking into account the above mentioned mechanism of fiber movement in the sampling zone, to describe the process of the dynamic interaction of individual fibers with the teeth of the headset, we accept the following calculation schemes.

\section{The Main Part}

We model the fiber capture pattern with the front face of the tooth, along which it makes a movement, and thus affects the process of pulling it out of the pulp (tape). A number of studies of domestic and foreign scientists are devoted to the modeling of such processes [1]-[9].

The arrangement of a fiber of mass $m$ on the tooth surface is shown in Figure 1 , Where $\alpha$ denotes the angle between the front face of the tooth and the direction of the radius-the $O B$ vector. We denote the position of the point $M$ along the tooth by the distance $r=B M$.

In Figure 1. angles $\beta, \delta u$ and $\gamma$ will be equal

$$
\beta=\omega \cdot t-\varphi_{0} ; \gamma=\delta=\alpha+\omega \cdot t-\varphi_{0}
$$

The coordinates of the material point of position (mass) $M(x, y)$ are calculated by the formulas

$$
\begin{aligned}
& x=O A+A C=R \cos \beta+r \cos \gamma=R \cos \left(\omega t-\varphi_{0}\right)+r \cos \left(\alpha+\omega t-\varphi_{0}\right) \\
& y=O A_{1}+A_{1} C_{1}=R \sin \beta+r \sin \gamma=R \sin \left(\omega t-\varphi_{0}\right)+r \sin \left(\alpha+\omega t-\varphi_{0}\right)
\end{aligned}
$$

We choose the distance $r=B M$ of the generalized coordinate and find the kinetic energy of a material point of mass $m$ by the formula:

$$
\begin{aligned}
T= & \frac{m}{2}\left(\dot{x}^{2}+\dot{y}^{2}\right) \\
= & \frac{m}{2}\left\{\left[-\omega R \sin \left(\omega t-\varphi_{0}\right)-r \omega \sin \left(\alpha+\omega t-\varphi_{0}\right)+\dot{r} \cos \left(\alpha+\omega t-\varphi_{0}\right)\right]^{2}\right. \\
& \left.+\left[\omega R \cos \left(\omega t-\varphi_{0}\right)+r \omega \cos \left(\alpha+\omega t-\varphi_{0}\right)+\dot{r} \sin \left(\alpha+\omega t-\varphi_{0}\right)\right]^{2}\right\} \\
= & \frac{m}{2}\left(\omega^{2} R^{2}+2 \omega^{2} r R \cos \alpha+2 \omega R \dot{r} \sin \alpha+r^{2} \omega^{2}+\dot{r}^{2}\right)
\end{aligned}
$$




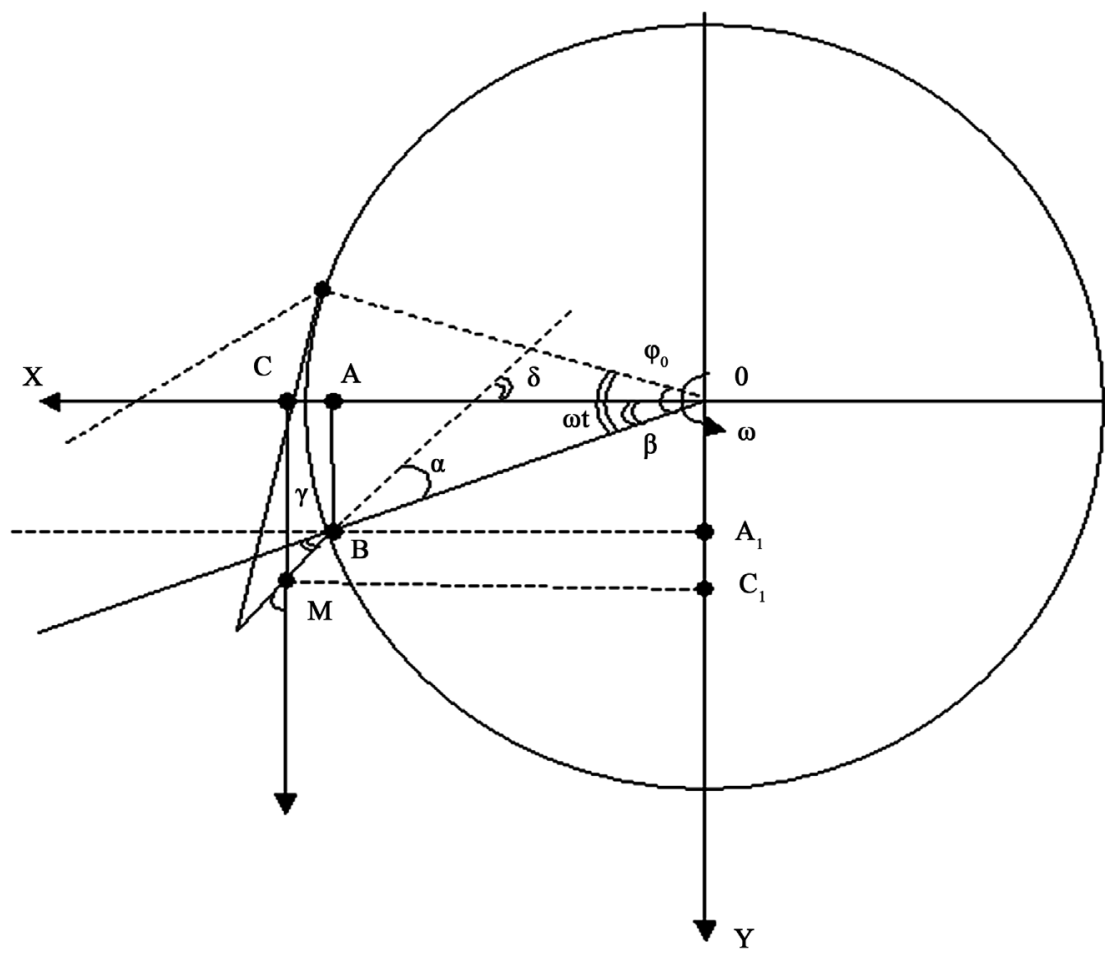

Figure 1. Layout of a fiber of mass $m$ on the tooth surface.

The mass is affected by the gravity of the material point and the friction force created by the force of gravity and the Carioles' force, the projections along the MV line will be equal.

$$
F_{g}=m g \sin \gamma ; \quad F_{g m p}=f m g \cos \gamma ; F_{k m p}=2 m f \omega \dot{r} \cos \alpha
$$

We now compose the second-order Lagrange equations

$$
\frac{\mathrm{d}}{\mathrm{d} t}\left(\frac{\sigma T}{\partial r}\right)-\frac{\sigma T}{\partial r}=F_{g}-F_{g m p}-F_{\kappa m p}
$$

Substituting the expression $T, F_{g}, F_{g t r}, F_{c t r}$ from (3) in (2.10) we obtain the equation for determining the distance $\mathrm{r}$ :

$$
\begin{aligned}
& \ddot{r}+2 f \omega \cos \alpha \cdot \dot{r}-\omega^{2} r \\
& =\omega^{2} R \cos \alpha+g\left[\sin \left(\alpha+\omega t-\varphi_{0}\right)-f \cos \left(\alpha+\omega t-\varphi_{0}\right)\right]
\end{aligned}
$$

Equation (5) is integrated under zero initial conditions:

$$
r=0 ; \dot{r}=0 \text { при } t=0
$$

The solution of Equation (6) is presented in the form:

$$
r=A_{1} \mathrm{e}^{\lambda_{1} \omega t}+A_{2} \mathrm{e}^{\lambda_{2} \omega t}+A_{0}+B_{0} \sin \left(\omega t+\alpha-\varphi_{0}\right)+C_{0} \cos \left(\omega t+\alpha-\varphi_{0}\right)
$$

where $\lambda_{1}=\sqrt{f^{2} \cos ^{2} \alpha+1}-f \cos \alpha, \lambda_{2}=-\sqrt{f^{2} \cos ^{2} \alpha+1}-f \cos \alpha$.

We substitute (7) into Equation (6) and equate the coefficients with constant and functions $\sin \left(\alpha+\omega t-\varphi_{0}\right)$ and $\cos \left(\alpha+\omega t-\varphi_{0}\right)$ to the right and left of the equality, then we get 


$$
A_{0}=-R \cos \alpha, \quad B_{0}=-\frac{g\left(1-f^{2} \cos \alpha\right)}{2 \omega^{2}\left(1+f^{2} \cos ^{2} \alpha\right)}, \quad C_{0}=-\frac{g f(1+\cos \alpha)}{2 \omega^{2}\left(1+f^{2} \cos ^{2} \alpha\right)},
$$

To determine the constants $A_{1}$ and $A_{2}$, we use the initial conditions (7), which give:

$$
\begin{aligned}
A_{1}+A_{2} & =-A_{0}-B_{0} \sin \left(\alpha-\varphi_{0}\right)-C_{0} \cos \left(\alpha-\varphi_{0}\right)+\lambda_{1} \omega A_{1}+\lambda_{2} \omega A_{2} \\
& =-B_{0} \omega \cos \left(\alpha-\varphi_{0}\right)+C_{0} \sin \left(\alpha-\varphi_{0}\right)
\end{aligned}
$$

From the last system we find the constants $A_{1}$ and $A_{2}$ :

$$
\begin{aligned}
& A_{1}=\frac{\lambda_{2} A_{0}+\left(\lambda_{2} B_{0}+C_{0}\right) \sin \left(\alpha-\varphi_{0}\right)+\left(\lambda_{2} C_{0}-B_{0}\right) \cos \left(\alpha-\varphi_{0}\right)}{\lambda_{1}-\lambda_{2}} \\
& A_{2}=\frac{\lambda_{1} A_{0}+\left(\lambda_{1} B_{0}+C_{0}\right) \sin \left(\alpha-\varphi_{0}\right)+\left(\lambda_{1} C_{0}-B_{0}\right) \cos \left(\alpha-\varphi_{0}\right)}{\lambda_{2}-\lambda_{1}}
\end{aligned}
$$

The movement of the fiber along the $B M$ line starts from the moment it is captured by the front face of the tooth, if the condition

$\ddot{r}>0$ at $t=0$ putting in the Equation (6) $\mathrm{z}$, we find

$$
\omega^{2} R \cos \alpha+g\left[\sin \left(\alpha-\varphi_{0}\right)-f \cos \left(\alpha-\varphi_{0}\right)\right] \geq 0
$$

or

$$
\omega^{2} \cos \alpha+g \cos \alpha\left[\operatorname{tg}\left(\alpha-\varphi_{0}\right)-f\right] \geq 0
$$

If you assume

$$
\alpha>\varphi_{0}, \quad \alpha<\frac{\pi}{2}, \quad \alpha-\varphi_{0}<\frac{\pi}{2},
$$

then the last inequality is always satisfied, if required $\operatorname{tg}\left(\alpha-\varphi_{0}\right)>f$ or $\alpha>\varphi_{0}+\operatorname{arctg} f$. If $\operatorname{tg}\left(\alpha-\varphi_{0}\right)<f$, or $\varphi_{0}<\alpha<\varphi_{0}+\operatorname{arctg} f$, then the condition of the drum speed satisfies the inequality

$$
\omega^{2}>\frac{g \cos \left(\alpha-\varphi_{0}\right)}{R \cos \alpha}\left(f-\operatorname{tg}\left(\alpha-\varphi_{0}\right)\right)
$$

Let now

$$
\alpha<\varphi_{0}, \quad \alpha<\frac{\pi}{2}, \varphi_{0}-\alpha<\frac{\pi}{2} .
$$

Then we rewrite inequality (9):

$$
\omega^{2} \cos \alpha-g \cos \left(\varphi_{0}-\alpha\right)\left[f+\operatorname{tg}\left(\varphi_{0}-\alpha\right)\right] \geq 0
$$

From the last inequality we have

$$
\omega^{2}>\frac{g \cos \left(\varphi_{0}-\alpha\right)\left(f+\operatorname{tg}\left(\varphi_{0} \alpha\right)\right)}{R \cos \alpha}
$$

If the angular velocity of the drum $\omega$ does not satisfy inequalities (10) and (11), then the movement of the fiber along the face of the tooth begins from the time determined from the equation

$$
\omega^{2} R \cos \alpha+g\left[\sin \left(\alpha+\omega t_{0}-\varphi_{0}\right)-f \cos \left(\alpha+\omega t_{0}-\varphi_{0}\right)\right]=0
$$


where, depending on the values $\omega$, $\alpha$ or $\varphi_{0}$ we find $t_{0}=0$ if $\omega^{2} \succ \frac{g \sqrt{1+f^{2}}}{R \cos \alpha}$,

$$
t_{0}=\frac{1}{\omega}\left[\beta_{0}+\varphi_{0}-\alpha-\arcsin \frac{\omega^{2} R \cos \alpha}{g \sqrt{1+f^{2}}}\right]
$$

at

$$
\omega^{2}<\frac{g \sqrt{1+f^{2}}}{R \cos \alpha} \quad \alpha<\varphi_{0}+\beta-\arcsin \frac{\omega^{2} R \cos \alpha}{g \sqrt{1+f^{2}}}
$$

where, $\beta_{0}=\arcsin \frac{1}{\sqrt{1+f^{2}}}$.

If we denote by $t_{k}$ the time spent by the fiber in the sampling zone, then the fiber moves along the effects of the tooth, if the conditions are met:

$$
r\left(t_{k}\right) \leq r_{0}
$$

where $r_{0}$-the length of the front edge of the tooth.

If condition (12) is not satisfied, then the fiber can leave the tooth surface before it enters the transportation zone.

Now we simulate the process of dragging the fiber with the teeth of the headset in the sampling zone. We accept that fibers as a deformable thread of finite length have an arcuate shape, the surface of which interacts with the surrounding pulp. When leaving the feed zone, all fiber points have a speed and a tension of $T_{0}$.

We accept that the fiber is deformed according to Hooke's law:

$$
T=E S \cdot \varepsilon
$$

where $E$-modulus, $S$-cross-sectional area of the fiber, is the relative deformation. If we denote by $V_{0}$ and $V$ the initial and current volume of the fiber, then the relative change in its volume will be equal

$$
\varepsilon_{\vartheta}=\frac{V-V_{0}}{V_{0}}
$$

\section{Moreover}

$$
\varepsilon_{\vartheta}=\varepsilon_{1}+\varepsilon_{2}+\varepsilon_{3}
$$

where $\varepsilon_{1}, \varepsilon_{2}, \varepsilon_{3}$-changes in the size of the thread in the axial and transverse directions. If we consider a thread of cylindrical shape, то $\varepsilon_{2}=\varepsilon_{3}=\mu \cdot \varepsilon \quad \mu$ Poisson. In view of the last equality, we have

$$
\varepsilon_{\vartheta}=(1+2 \mu) \varepsilon
$$

Then from Formula (14) we find

$$
\ddot{\varepsilon}=\frac{1}{1+2 \mu} \cdot \frac{V_{0}-V}{V_{0}} .
$$

On the other hand

$$
V_{0}=\frac{1}{\rho_{0}}, \quad V=\frac{1}{\rho},
$$


where $\rho_{0}$ and $\rho$-initial (when leaving the feed zone) and the current density of a single fiber, thus we have

$$
\varepsilon=\frac{1}{1+2 \mu} \cdot \frac{-\frac{1}{\rho_{0}}+\frac{1}{\rho}}{\frac{1}{\rho_{0}}}=\frac{\rho_{0}-\rho}{\rho}=\left(\frac{\rho_{0}}{\rho}-1\right) \frac{1}{1+2 \mu}
$$

From the last equality we express the change in density through deformation $\frac{\rho_{0}}{\rho}=(1+2 \mu) \varepsilon+1$.

Formula (13) is written as

$$
T=\frac{E}{1+2 \mu}\left(\frac{\rho_{0}}{\rho}-1\right) S
$$

The process of dragging the fiber is considered stationary; in this case, the law of conservation of mass

$$
\rho_{0} S_{0} \vartheta_{0}=\rho S \vartheta
$$

Here $S_{0}$ and $S$ fiber cross section, $\vartheta$-section speed. From the Equation (15) taking into account (16), half

$$
\frac{\vartheta}{\vartheta_{0}}=\frac{\rho_{0}}{\rho} \cdot \frac{S_{0}}{S}=\frac{S_{0}}{S}\left[1+\frac{T(1+2 \mu)}{E S}\right]
$$

In the future we get $S_{0}$ and $S$ we have put

$$
\vartheta=\vartheta_{0}\left[1+\frac{T(1+2 V)}{E S_{0}}\right]
$$

Accordingly, for density we have:

$$
\frac{\rho}{\rho_{0}}=\frac{\vartheta_{0}}{\vartheta}=\frac{1}{1+\frac{T(1+2 \mu)}{E S_{0}}}
$$

Formulas (17) and (18) establish the relationship between the tension of the thread and the speed as well as the density in an arbitrary section of the fiber.

Let us create the equation of the balance of the thread taking into account the resistance of its environment [10] [11]:

$$
\frac{\mathrm{d} T}{\mathrm{~d} l}-\tau=0, \frac{T}{R}-q=0
$$

where, $l$ - the length of the arc to be reported from the point of the fiber exit from the feed zone; $\tau$ and $q$ intensity of tangential normal forces on the surface of the thread.: We accept, then the values $\tau$ and $q$ are bound according to the Kulon law by dependence $\tau=f \cdot q$ (f-coefficient of friction). With this in mind...

$$
\frac{\mathrm{d} T}{\mathrm{~d} l}-f \frac{T}{R}=0, \quad 0 \leq l \leq \omega t \cdot R
$$

where $\omega$ and $R$ the angular velocity and radius of the drum. We introduce the 
moving coordinate $x=l-\omega t \cdot R$ and integrate the Equation (20).

$$
T=A \mathrm{e}^{\frac{f l}{R}}
$$

where $A$ is an arbitrary constant determined from the condition of capturing (dragging) the fiber by the teeth of the headset.

$$
\vartheta=\omega \cdot R \text { при } x=0
$$

Then from (2.23) we find the tension value in the section $S=0$

$$
T_{0}=\left(\frac{\omega R}{\vartheta_{0}}-1\right) \frac{E S_{0}}{1+2 \mu}
$$

Taking into account (22), the Formula (21) takes the form

$$
T=\left(\frac{\omega R}{V_{0}}-1\right) \frac{E S_{0}}{1+2 V} \mathrm{e}^{f \frac{l-\omega t}{R}}
$$

The speed and density in any section is calculated by formulas:

$$
\begin{gathered}
V=V_{0}\left[1+\left(\frac{\omega R}{\vartheta_{0}}-1\right) \mathrm{e}^{f \frac{l-\omega t}{R}}\right] \\
\rho=\rho_{0} \frac{1}{1+\left(\frac{\omega R}{\vartheta_{0}}-1\right) \mathrm{e}^{f \frac{l-\omega t}{R}}}
\end{gathered}
$$

\section{Analysis of the Results}

The obtained equations are processed on a computer using the Maple program with the natural parameters of technological machines and graphical dependencies are obtained fiber movement when moving along the tooth of the headset at different angles from time to time (Figure 2); the distribution of fiber tension along the arc of the sampling zone at different speeds of rotation of the drum (Figure 3), the distribution of fiber speed along the arc of the sampling zone at different speeds of rotation of the drum (Figure 4), according to which it can be noted that all the dependencies have an upward character of change .

The dependence of the movement of the fiber when moving along the tooth of the headset at different angles from time to time (Figure 2) also shows that, in hundredths of a second ( $0.001 \mathrm{sec})$, the movement of the fiber does not exceed 3 $-7 \mathrm{~mm}$ at angles- $\alpha=10^{\circ}, \alpha=30^{\circ}, \alpha=45^{\circ}, \alpha=60^{\circ}$.

A, the dependence of the distribution of fiber tension along the arc of the sampling zone at different speeds of rotation of the drum (Figure 3 ) shows that at angular speeds $600-750 \mathrm{sek}^{-1}$, a change in distance from 0 to $0.1 \mathrm{~m}$ leads to an increase in fiber tension in the range $19-24 \mathrm{cN}$.

Also, the dependence of the distribution of the fiber velocity along the arc of the sampling zone at different speeds of rotation of the drum (Figure 4) shows that at angular speeds of $600-750 \mathrm{sec}^{-1}$, a change in the distance from 0 to $0.1 \mathrm{~m}$ contributes to an increase in the fiber speed from $37-49 \mathrm{~m} / \mathrm{s}$. 


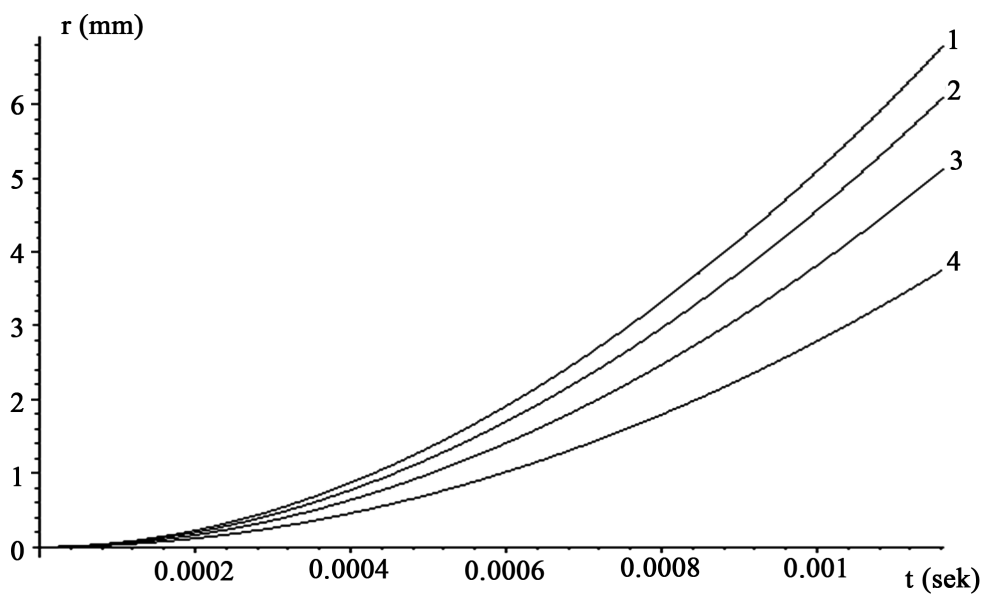

Figure 2. The time-dependant $t$ (сек) movement of the fiber $r($ мм) along the headset tooth at different angles $\alpha 1-\alpha=10^{\circ}, 2-\alpha=30^{\circ}, 3-\alpha=45^{\circ}, 4-\alpha=60^{\circ}$.

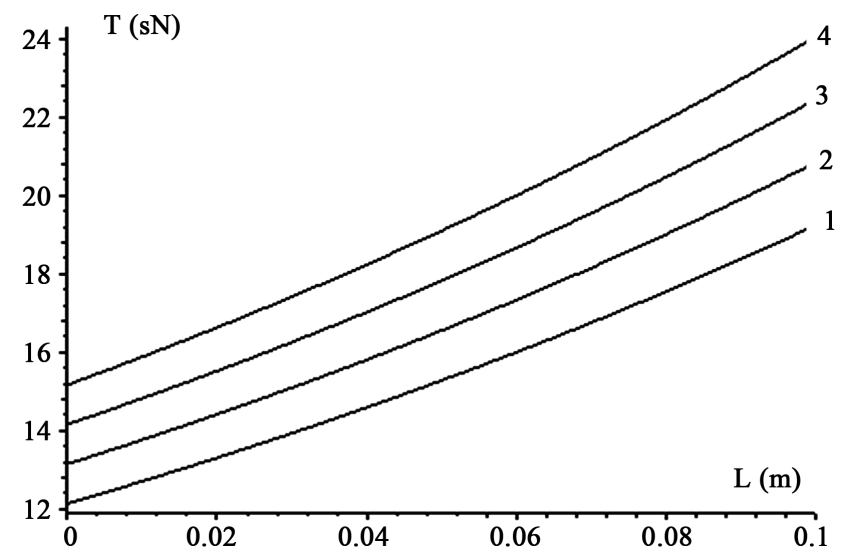

Figure 3. Distribution of fiber tension $T(\mathrm{cH})$ along the arc of the sampling zone at different rotational speeds of the drum $\omega(1 / \mathrm{sec}) 1-\omega=600,2-\omega=650,3-\omega=700,4$ $\omega=750$.

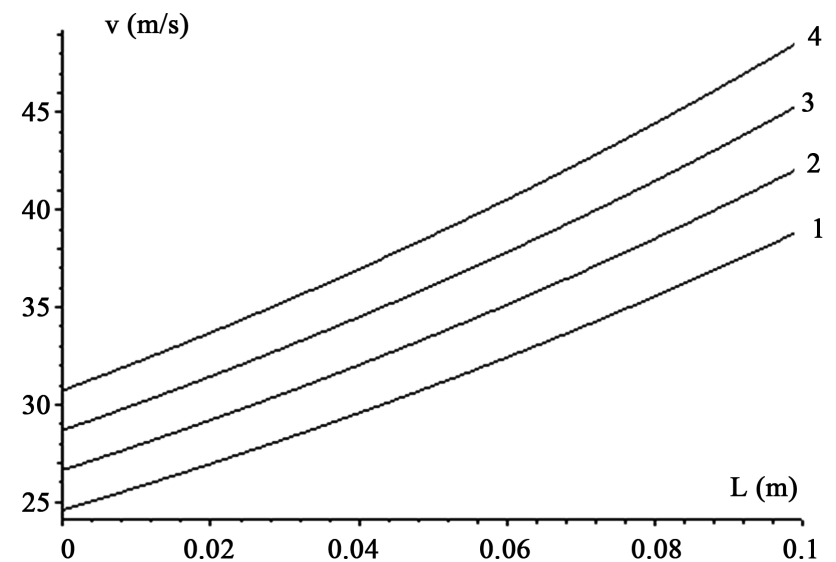

Figure 4. Distribution of fiber velocity $v(\mathrm{M} / \mathrm{c})$ along the arc of the sampling zone at different rotational speeds of the drum $(1 / \mathrm{sec}) .1-\omega=600,2-\omega=650,3-\omega=700,4$ $\omega=750$. 


\section{Conclusions}

1) The regularity was studied and the equation of fiber motion on the tooth surface of the headset of the sampling drum was obtained.

2) As a result of the analysis of the tension and speed of the fibers, it was found that with an increase in the sampling zone, the tension force and the speed of the fiber increase.

\section{Conflicts of Interest}

The authors declare no conflicts of interest regarding the publication of this paper.

\section{References}

[1] Roglin, B., Bouzhek, A., Wilfert, M., et al. (1981) Spinning-Free Spinning. Light and Food Industry, 86-121.

[2] Yuldashev, Zh. (2011) Improving the Quality of Pneumomechanical Yarn Using Multi-Thread Winding of the Headset. Dissertation of the Candidate of Technical Sciences, Tashkent Institute of Textile and Light Industry, Tashkent, Uzbekistan, 52-85.

[3] Abdukarimovich, M.O., Ibragimovich, A.K. and Sharipjanovich, S.O. (2018) Designing a New Design of a Loading Cylinder for Pneumomechanical Spinning Machines. Engineering, 10, 345-356. https://doi.org/10.4236/eng.2018.106025

[4] Abbazov, I., Sarimsakov, O., Khodjiev, M. and Mardonov, B. (2018) Waste Produced at Cotton Waste Factories. American Journal ASCIT Communications, 5, $22-28$.

[5] Kholmirzaev, F., Azimov, S., Abdurahimov, K. and Sarimsakov, O. (2019) Investigation of the Loss of Air Pressure in the Pipeline of the Cotton Pneumatic Conveying. Saudi Journal of Engineering and Technology, 4, 23-27.

[6] Muradov, R., Burnashev, R.Z. and Sarimsakov, O.Sh. (2001) The Dynamic Task of the Interaction of Raw Cotton with the Working Bodies of Processing Machines. Journal of Mechanic Problems, No. 34, 55-58.

[7] Obidov, A., Akhmedkhodjaev, Kh., Sarimsakov, O. and Holikov, Q. (2018) Investigation of the Properties of Fibrous Cotton Seeds, for Sorting on a Mesh Surface. Engineering, 10, 572-578. https://doi.org/10.4236/eng.2018.109041

[8] Sarimsakov, O. (2016) The Possibility of Reducing Cotton Consumption in Cotton. American Journal of Science and Technology, 4, 68-72.

[9] Sarimsakov, O. (2019) Improving the Process of Filing Cotton in Pneumatic Transport and its Transportation by Air. Monography, Namangan, Uzbekistan, p. 252.

[10] Sarimsakov, O. and Gaybnazarov, E. (2017) About Energy Consumption in Pneumatic Conveying of Raw Cotton. American Journal of Energy and Power Engineering, 3, 26-29.

[11] Sarimsakov, O., Xusanov, C. and Muradov, R. (2016) The Change in Air Pressure along the Length of the Pipeline Installation for Pneumatic Conveying of Raw Cotton. Journal of Engineering and Technology, 3, 89-92. 